\title{
Anomalous Absorption During Artificial Modification at Harmonics of the Electron Gyrofrequency
}

\author{
A. J. STOCKER, F. HONARY, T. R. ROBINSON, AND T. B. JONES \\ Department of Physics and Astronomy, Leicester University, Leicester, England
}

P. STUBBE

Max-Planck-Institut fur Aeronomie, Lindau, Germany

\begin{abstract}
Observations of the anomalous absorption of low-power HF probe waves caused by the action of a highpower HF ordinary mode radio wave are presented. At pump frequencies in the vicinity of harmonics of the electron gyrofrequency the anomalous absorption measured on the low-power probe waves exhibit local minima. Also at these frequencies, large-scale changes deduced from phase measurements of the low-power probe waves and heater self-absorption are reduced, whereas the growth time of the anomalous absorption is increased. The electron gyrofrequency estimated from the frequency of the absorption minimum compares favorably with that derived from the International Geomagnetic Reference Field (IGRF) magnetic field model.
\end{abstract}

\section{INTRODUCTION}

Anomalous or wideband absorption [e.g., Cohen and Whitehead, 1970; Utlaut and Violette, 1974; Stubbe et al., 1982a; Jones et al., 1984, 1986; Robinson, 1989; Stocker et al., 1992a] has been a common feature of experiments at all geomagnetic latitudes in which a powerful HF radio wave of ordinary polarization interacts with the plasma in the ionospheric $F$ region. The anomalous absorption of low-power probe $H F$ radio waves propagating through the reflection region of a high power pump wave arises because of the generation of small-scale field-aligned irregularities (FAI) by the pump in the vicinity of the upper hybrid resonance region [Perkins, 1974; Vas'kov and Gurevich, 1975, 1977; Graham and Fejer, 1976; Das and Fejer, 1979; Stubbe and Kopka, 1980; Kuo and Lee, 1982; Stubbe et al., 1982a, b; Dysthe et al., 1983; Jones et al., 1984, 1986; Robinson, 1988, 1989]. Predominantly, the probe waves are scattered into Langmuir waves rather than into other electromagnetic waves [Ryzhov, $1971,1972]$. Heater-induced anomalous absorption has been studied extensively with the aid of the Max-Planck-Institut (MPI) high-power facility (heater) at Troms $\emptyset$, Norway [Stubbe et al., 1982a; Jones et al., 1982, 1984, 1986; Robinson, 1989]. In these previous experiments the heater was operated at arbitrarily chosen convenient frequencies. Recently, however, there has been a great deal of interest in the effects of heating at frequencies close to harmonics of the electron gyrofrequency [Leyser et al., 1989, 1990, 1992; Leyser, 1991; Stubbe and Kopka, 1990; Belyakova et al., 1991; Lobachevsky et al., 1992]. So far, all of these experiments except those reported by Belyakova et al. [1991] and Lobachevsky et al. [1992] have concentrated on observations of the spectrum of the reflected heater signal, i.e. the so-called stimulated electromagnetic emission (SEE) spectra [Thidé et al., 1982, 1983, 1989; Stubbe et al., 1984; Fejer et al., 1985]. The downshifted maximum (DM) feature observed in the SEE

Copyright 1993 by the American Geophysical Union.

Paper number 93JA00878.

0148-0227/93/93JA-00878\$05.00 spectra [Stubbe et al., 1984] has been associated with the existence of FAI and is found not to be present at pump frequencies near gyrofrequency harmonics [Leyser et al., 1990]. Belyakova et al. [1991] and Lobachevsky et al. [1992] studied Doppler measurements made during heating. They found that while small-scale electron density perturbations were found near the pump reflection and upper hybrid resonance (UHR) heights during heating far from gyrofrequency harmonics, during heating at the third harmonic these perturbations were not detected at the UHR. The disappearance of the DM at gyrofrequency harmonics and its apparent dependence on the presence of FAI prompted the investigation of anomalous absorption characteristics at pump frequencies close to gyrofrequency harmonics which is reported in this paper.

Observations from two campaigns (May and August 1991) in which the signal strength and phase of low-power HF diagnostics and heater self-absorption were measured at heater frequencies close to harmonics of the electron gyrofrequency are presented in section 3 . In section 4 values of the electron gyrofrequency estimated from measurements of the anomalous absorption are compared with those derived from the IGRF model, and finally, possible explanations for some of the observed features are also discussed.

\section{EXPERMMENTAL ARRANGEMENT}

The experimental arrangement for the present experiment is almost identical to that employed for several previous observations of anomalous absorption at Troms $\varnothing$ [e.g., Jones et al., 1986]. The locations of the various sites where equipment was deployed in two heating campaigns (May and August 1991) are illustrated in Figure 1. The heater which is located at Ramfjordmoen about $16 \mathrm{~km}$ to the southeast of Troms $\varnothing$, Norway, has been described by Stubbe et al. [1982b]. However, recently, the number of elements in one of the antenna arrays has been increased by a factor of 4 resulting in an increased gain of $6 \mathrm{~dB}$. Thus a maximum effective radiated power (ERP) of about $1 \mathrm{GW}$ can now be transmitted in the frequency range $5.5-8 \mathrm{MHz}$ (the so-called "superheater"). During the experiments described in this paper, the beater 


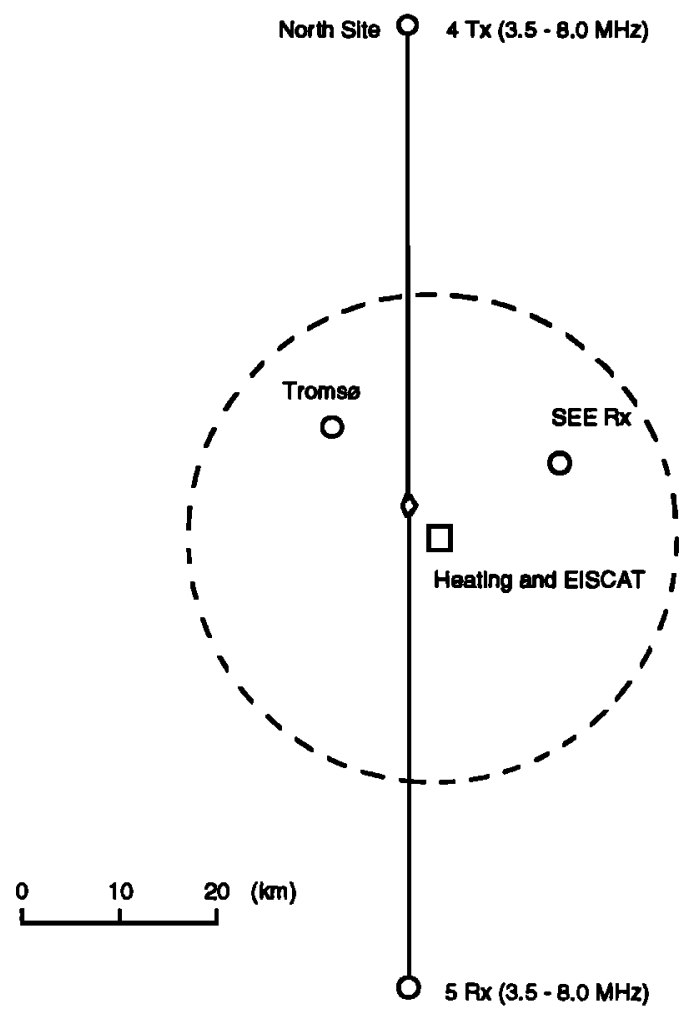

South Site

Fig. 1. Map showing the location of the experimental stations. The dashed circle indicates the approximate position of the -3-dB contour of the lower-power (i.e., wider beam) heater at an altitude of $200 \mathrm{~km}$ (note that the effects of refraction have been neglected).

transmitted vertically with $o$-mode polarization from a lowergain antenna array (i.e., ERP 160-200 MW) with the exception of an experiment on May 15, 1991, which employed the higher-gain, superbeater antenna array (ERP $640 \mathrm{MW}$ ). Given that during periods of low $D$ region absorption the threshold of the thermal parametric instability, which leads to FAI growth and hence anomalous absorption, is easily exceeded by pump waves transmitted from either array [e.g. Stubbe and Kopka, 1980; Robinson, 1989], no systematic difference in the anomalous absorption measurements should be expected.

Low-power HF transmitters were located $50 \mathrm{~km}$ to the north of the heater site, while the receivers were positioned $45 \mathrm{~km}$ to the south. This geometry was chosen deliberately in order to ensure that the probe waves would not propagate through the heated $D$ or $E$ regions. This diagnostic system consists of four transmitters capable of broadcasting unmodulated signals in the range $3.5-8 \mathrm{MHz}$ with a power of about $50 \mathrm{~W}$. The signals are then radiated from inverted- $V$ antennas which induce both $o$ and $x$ modes of propagation in the ionosphere. These transmissions are received on crossed dipole antennas which enables the polarization modes to be resolved and recorded separately. The real and imaginary components of the received signals are logged which means that both phase and amplitude information can be obtained. The data are sampled at a rate of $32 \mathrm{~Hz}$ and written to magnetic tape. Simultaneously, the signal amplitudes are also written to chart recorders so that a real-time assessment of heating effects can be made during the course of the experiments.

As well as the diagnostics described above, a further HF receiver was located at a site $17 \mathrm{~km}$ to the northeast of the heater where the spectrum of the reflected heater wave and hence the SEE were measured [Stubbe et al., 1984]. The results of observations obtained by this equipment during the present two campaigns will be reported elsewhere (P. Stubbe et al., Stimulated electromagnetic emissions and anomalous absorption near harmonics of the electron gyrofrequency, submitted to Journal of Geophysical Research, 1993). Ionograms produced by an ionosonde collocated with the heater were recorded on film at approximately 30 to $50 \mathrm{~min}$ intervals and thus provided a general indication of ionospheric background conditions. The ambient electron density profiles and hence heater interaction heights were calculated from these ionograms (section 4).

Although the experiments during the two campaigns were similar, there were some differences which are outlined below. A schematic of the heater cycles employed during these experiments is depicted in Figure 2. The heater was first tuned to the required frequency, a process which usually took 1 to 2 min. Then, during a 4 min period when the heater was operating, the spectrum of the reflected heater signal was measured. In the May 1991 experiments this was followed by two heater cycles of $30 \mathrm{~s}$ off, followed by $30 \mathrm{~s}$ on. In August 1991, one heater cycle of $1 \mathrm{~min}$ off, followed by $1 \mathrm{~min}$ on, was transmitted. The first scheme (May 1991) enabled good estimates of heater self-absorption and the onset time of the anomalous absorption to be obtained, while the second scheme (August 1991) provided improved estimates of the anomalous absorption. The heater was then switched off for about $1 \mathrm{~min}$ before being tuned to the next frequency and the experimental procedure repeated. The heater frequency was changed by either 10 or $20 \mathrm{kHz}$ at each step, over a total range of typically 300 kHz. Each experiment began at a frequency between 80 and 150 $\mathrm{kHz}$ below the expected value of the harmonic of the electron gyrofrequency and progressed upward through the harmonic and beyond. Given sufficient time and favorable ionospheric conditions a second run was usually made in which the frequency was stepped downward through the harmonic. In this way an experiment took about 3 to 4 hours to complete and therefore very stable conditions were required for systematic measurements to be obtained.

Throughout the heater cycle, low-power HF probe waves were received at the south site. In May 1991, three diagnostic signals were monitored: two at fixed frequencies, one about 0.5 MHz below the harmonic of the gyrofrequency ( $O$ mode), the other about $0.5 \mathrm{MHz}$ above (both $o$ and $x$ modes), and one at a

a)

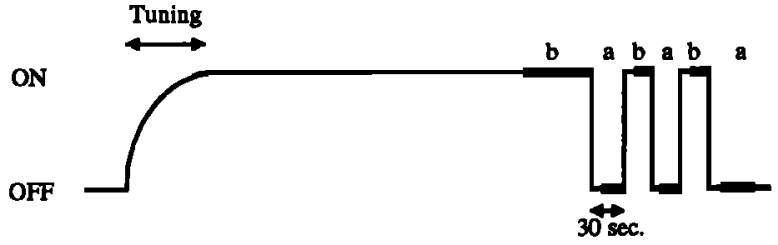

b)

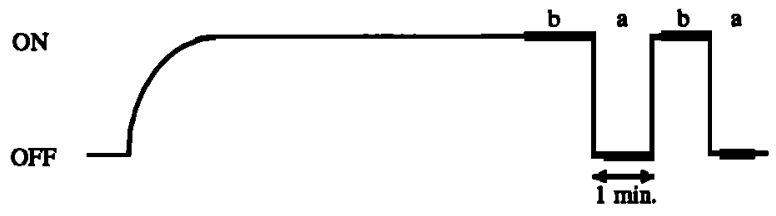

Fig. 2. A schematic of the heater cycles employed during (a) May 1991, and (b) August 1991. The thick lines ' $a$ ' and ' $b$ ' represent the intervals from which data were averaged to obtain $A_{\text {off }}$ and $A_{\text {on }}$, respectively. 
frequency which was offset from that of the heater by a constant value (in the region of $60 \mathrm{kHz}, o$ mode). Similarly, in August 1991, three diagnostic signals were measured but only one fixed frequency signal was employed, either $0.5 \mathrm{MHz}$ above or below the harmonic of the gyrofrequency. In the case of the fixed frequency being above the gyrofrequency harmonic, both $o$ and $x$ modes were monitored, otherwise only the $o$ mode was recorded. In addition to the fixed frequency signal, two diagnostics at a constant offset from the heater, one $70 \mathrm{kHz}$ below and one $70 \mathrm{kHz}$ above the heater frequency (both $O$ mode), were also monitored. The anomalous absorption measurements which are presented in this paper are derived from probe waves which were offset by a fixed frequency from the beater. In both campaigns the nonfixed frequency (i.e., those at a fixed frequency offset from the heater) receivers were switched off when the frequency of the probe transmitter was changed to provide a visual reference point in the data. The fixed frequency diagnostics provided a pointer to any changes in the ambient ionospheric conditions and also a rough indication of the FAI scale size [Jones et al., 1984; Robinson, 1989; Stocker et al., 1992a]. The amplitude of the reflected heater signal was also monitored during both campaigns.

\section{OBSERVATIONS}

\subsection{Diagnostic Anomalous Absorption}

A typical example of the signal strengths of two $o$-mode probe waves, one $70 \mathrm{kHz}$ above and one $70 \mathrm{kHz}$ below the heater frequency, as a function of time is illustrated in Figure 3. The amplitude of the reflected heater signal is reproduced in the bottom panel of this figure. This figure demonstrates quite clearly that the heater causes a reduction in the received strength of the low-power waves, i.e., anomalous absorption [e.g., Stubbe et al., 1982a; Robinson, 1989]. The level of anomalous absorption, $\Gamma$, can be defined quite simply as $\Gamma=$ $A_{\text {off }}-A_{\text {on }}$, where $A_{\text {off }}$ and $A_{\text {on }}$ are the quasi-stationary signal strengths observed while the heater was off and on, respectively. By "quasi-stationary" we mean that the signals are fluctuating about a relatively constant level. This is not the case, for example, immediately after the heater has been switched on or off or while the probe frequency is altered. Values of $A_{\text {off }}$ and $A_{\text {on }}$ for each pump frequency are estimated by averaging the data over the intervals marked $a$ and $b$, respectively, in Figure 2. In common with many other observations of this type at Troms $\varnothing$, the growth and recovery times of the absorption (Figure 3) are a few seconds (except the growth time observed by the 6.70-MHz diagnostic at 1349 UT) and the absorption levels recorded are about $10 \mathrm{~dB}$. Several other features present in Figure 3 also require comment. Firstly, it should be noted that during the tuning process of the heater (1342:10 - 1343:00 UT), the heater beam is not fully focused and therefore as a result the anomalous absorption of the diagnostic signals does not begin until after the end of the tuning period. The difficulty in determining the time at which the diagnostics begin to be anomalously absorbed means that the growth time of the anomalous absorption cannot be estimated with any confidence during this part of the heater cycle. Secondly the two sudden reductions in the probe signal strength which occur at approximately 1341:40 and 1350:40 UT are the visual reference points referred to in section 2 and therefore indicate that the diagnostic frequency was being changed.

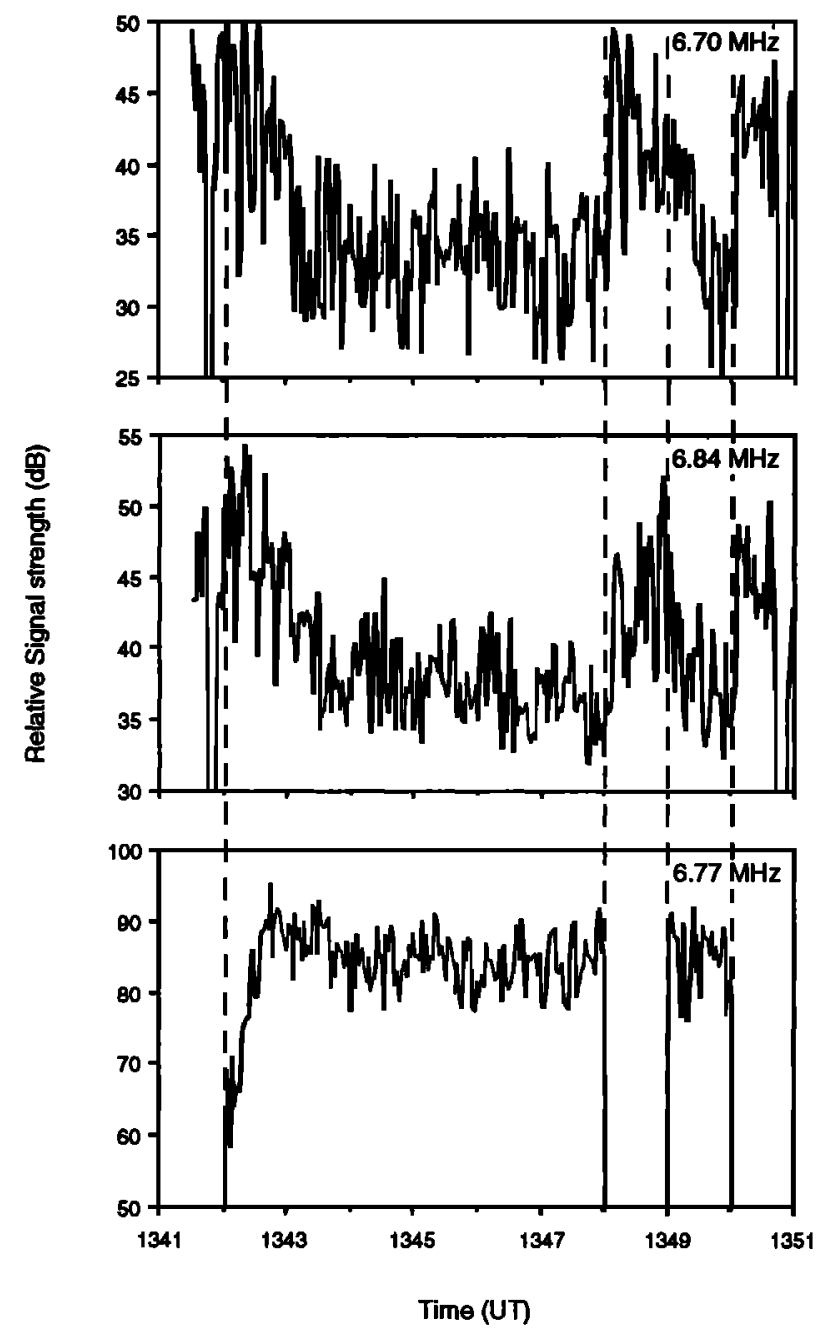

Fig. 3. The relative signal strength of the reflected heater wave (bottom panel) and two probe waves (top two panels) on August 25, 1991.

An example of the anomalous absorption response to different heater frequencies between 1452 and 1700 UT on August 24, 1991, is presented in Figure 4. During this interval the levels of anomalous absorption measured on the probe waves $70 \mathrm{kHz}$ above and below the heater were almost identical and therefore they have been averaged to produce the data in Figure 4. The error bars represent the standard error of the mean associated with the averaging of these data. It is clear from this figure that the anomalous absorption is reduced at frequencies close to $6.70-6.72 \mathrm{MHz}$. There is evidence to suggest that this frequency lies close to a harmonic of the electron gyrofrequency (see section 4). The "double dip" feature, i.e., the two local minima either side of the peak at $6.71 \mathrm{MHz}$, which is present in Figure 4 is observed during all experiments where the heater frequency is close to the fifth harmonic of the gyrofrequency and sometimes at other harmonics. Note that the level of anomalous absorption does not fall to zero even at the minimum.

In order to highlight the systematic features, values of the anomalous absorption observed in the vicinity of three different gyrofrequency harmonics (i.e., at $\mathrm{nf}_{\mathrm{c}}$, with $\mathrm{n}=3,4$, and 5 , and where $f_{c}$ is the electron gyrofrequency) have been averaged over a number of experiments and plotted as functions of heater frequency in Figure 5. The zero point in the frequency scale bas been chosen to coincide with the minimum in 


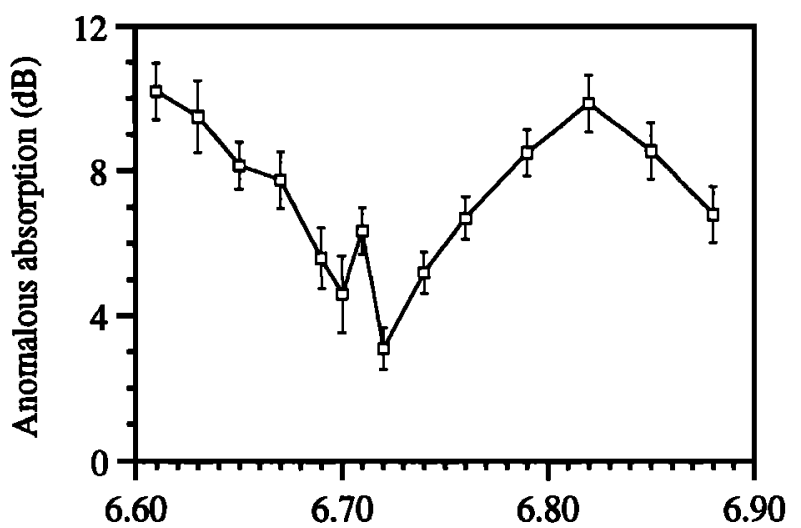

Heater frequency $(\mathrm{MHz})$

Fig. 4. The average of the anomalous absorption measured on two probe waves fixed at frequencies of $70 \mathrm{kHz}$ above and below the heater frequency as a function of heater frequency close to the fifth gyrofrequency harmonic for an experiment on August 24, 1991 (1452 1700 UT).
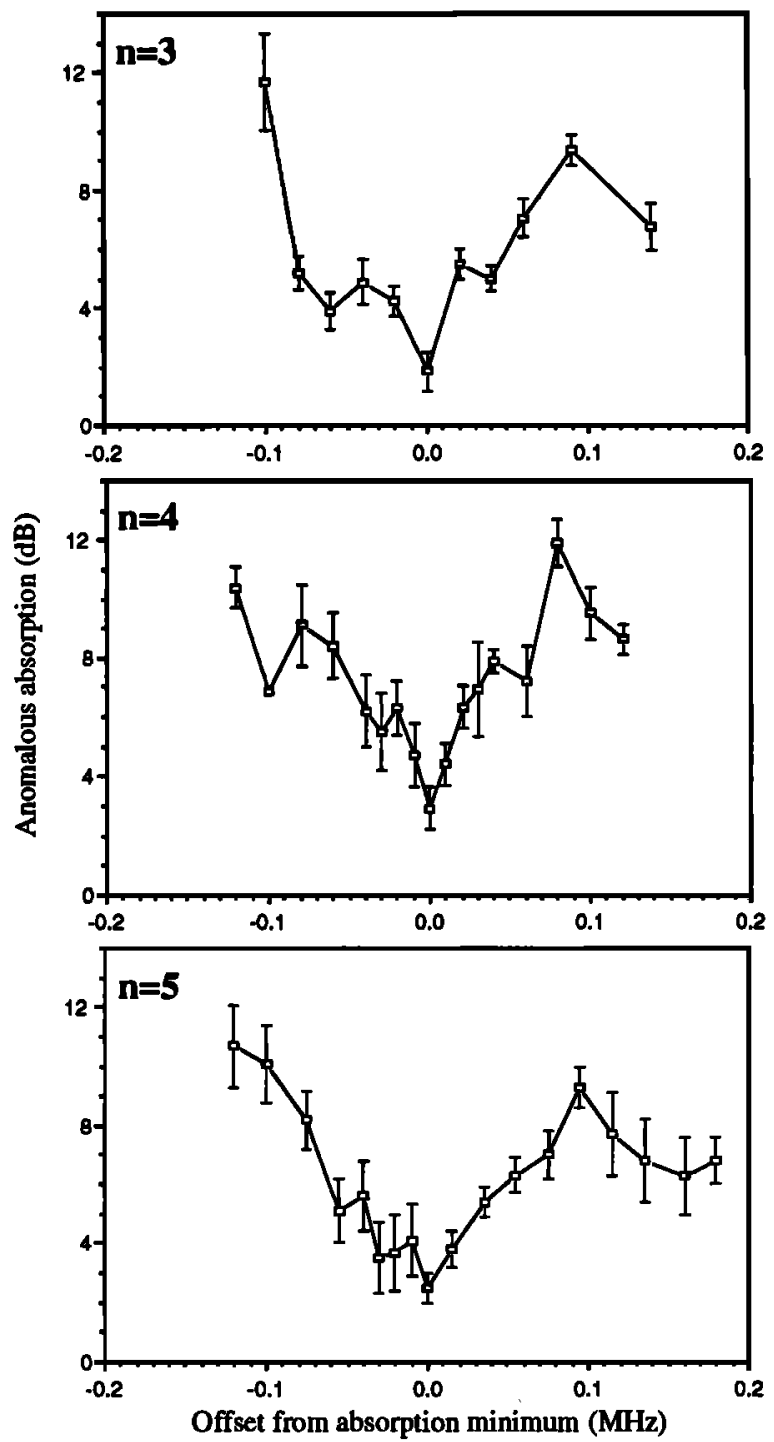

Fig. 5. Averaged anomalous absorption as a function of pump frequency offset from the minimum in anomalous absorption, $\Delta f$ for three gyrofrequency harmonics (i.e., $\mathrm{nf}_{\mathrm{c}}$ ). For $\mathrm{n}=3$, the results from only one experiment has been included in the average, whereas the results from six and five experiments were averaged for $n=4$ and $n=5$, respectively. anomalous absorption in each experiment. It should be pointed out that the minimum in anomalous absorption did not necessarily occur at the same frequency for each experiment, an observation which is hardly surprising if the location of the minimum is related to the electron gyrofrequency since this will vary with magnetic field strength and therefore heater reflection height. This point is discussed further in section 4. As in Figure 4, the most obvious feature at all three values of $n$ in Figure 5 is that the anomalous absorption reduces to a minimum of about $2-3 \mathrm{~dB}$ for a narrow range $(20-30 \mathrm{kHz})$ of pump frequencies. From measurements of the spectrum of the reflected heater wave, it appears that the minimum in anomalous absorption is related to the disappearance of the downshifted maximum feature in the stimulated emissions ( $P$. Stubbe, private communication, 1991). A peak in the anomalous absorption appears at $n=3,4$, and 5 for a heater frequency of about $80-100 \mathrm{kHz}$ above that of the minimum. Some trace of the "double dip" can also be observed in Figure 5 in the $n=4$ (at $-30 \mathrm{kHz}$ ) and $n=5$ (at -10 and $-40 \mathrm{kHz}$ ) ranges, although these features are similar in magnitude to the errors.

In addition to the level of diagnostic anomalous absorption, anomalous absorption growth time constant, and diagnostic phase changes have also been measured. The growth time of the anomalous absorption has been derived from the e-fold time of an exponential curve least squares fitted to the diagnostic signal level. The anomalous absorption, anomalous absorption growth time constant, and diagnostic phase changes obtained during an experiment on May 16, 1991, when the heater frequency was close to the fourth harmonic of the electron gyrofrequency, have been plotted as functions of heater frequency in Figure 6. The same quantities measured during heating close to the fifth harmonic ( $E R P=640 \mathrm{MW}$ ) on May 15, 1991, are illustrated in Figure 7. In addition to the quantities derived from observations of the diagnostic signals, the heater self-absorption has also been measured and is depicted as a function of pump frequency in Figures $6 d$ and $7 d$ and discussed in subsection 3.3.

Examination of Figures $6 a$ and $7 a$ reveals that the anomalous absorption as a function of heater frequency exhibits a local minimum, as discussed previously. In the case of the experiment on May 16, 1991 (Figure 6a), the anomalous absorption is reduced to less than $1 \mathrm{~dB}$ at a frequency of 5.443 MHz (i.e., $f_{c} \sim 1.361 \mathrm{MHz}$ ), while for May 15, 1991 (Figure 7a), the minimum in absorption occurs for a pump frequency of around 6.69 $\mathrm{MHz}$ (i.e., $\mathrm{f}_{\mathrm{c}} \sim 1.338 \mathrm{MHz}$ ). The difference in gyrofrequency measured in each of these experiments is consistent with a difference in the respective heater reflection heights (see section 4).

The e-fold time of the onset of diagnostic absorption as a function of heater frequency on May 16 is illustrated in Figure $6 b$. It appears from this figure that the e-fold time increases as the heater frequency approaches the harmonic of the electron gyrofrequency. However, the anomalous absorption is a minimum at frequencies in the vicinity of the harmonic of the electron gyrofrequency and therefore the e-fold time cannot be accurately determined since the natural variation in diagnostic signal strength (i.e., fading) can be of the same order as the heater-induced absorption. The growth time of anomalous absorption for a given set of ionospheric conditions, in particular variability [Stubbe et al., 1982a], is a crude measure of by how much the electric field of the heater wave exceeds the excitation threshold of the thermal parametric decay instability. Previous experiments [Robinson, 1989] indicated 

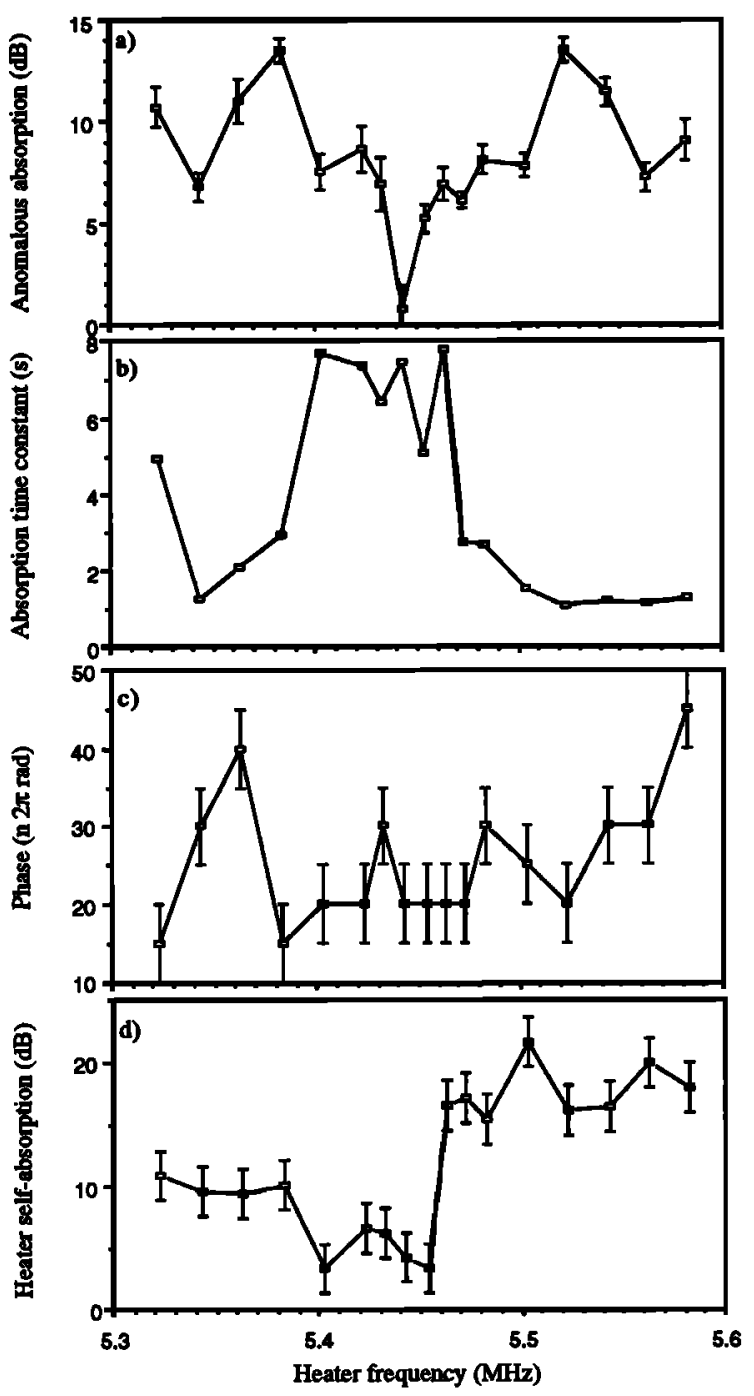

Fig. 6. (a) Anomalous absorption, (b) absorption growth time, (c) heaterinduced phase change, and (d) heater self-absorption, as a function of heater frequency close to the fourth gyrofrequency harmonic on May 16, 1991. Heater effective radiated power (ERP) $-200 \mathrm{MW}$.

that increasing heater power tended to reduce the growth time. So the observations presented in Figure $6 b$ may indicate that the heater power employed in creating FAI is reduced at frequencies close to gyrofrequency harmonics. The growth times measured on May 15 (Figure $7 b$ ) were seriously affected by natural signal fading and are therefore not as clearly related to the anomalous absorption as those on May 16 (Figure 6b).

\subsection{Diagnostic Phase}

In addition to the small-scale effects (i.e., the creation of FAI and hence anomalous absorption), large-scale or bulk effects are also known to occur during heating at Troms [ Jones et al., 1982, 1986; Robinson, 1989; Stocker et al., 1992b]. The phase change observed on the received diagnostic signals is a measure of these bulk changes since the phase path is dependent upon the refractive index along the signal path which in turn depends on the electron density. It is important to note that an increase in the measured phase in Figures 6 and 7 represents a decrease in the phase path between the transmitter and the receiver. The maximum phase change measured at each heater frequency is plotted as a function of

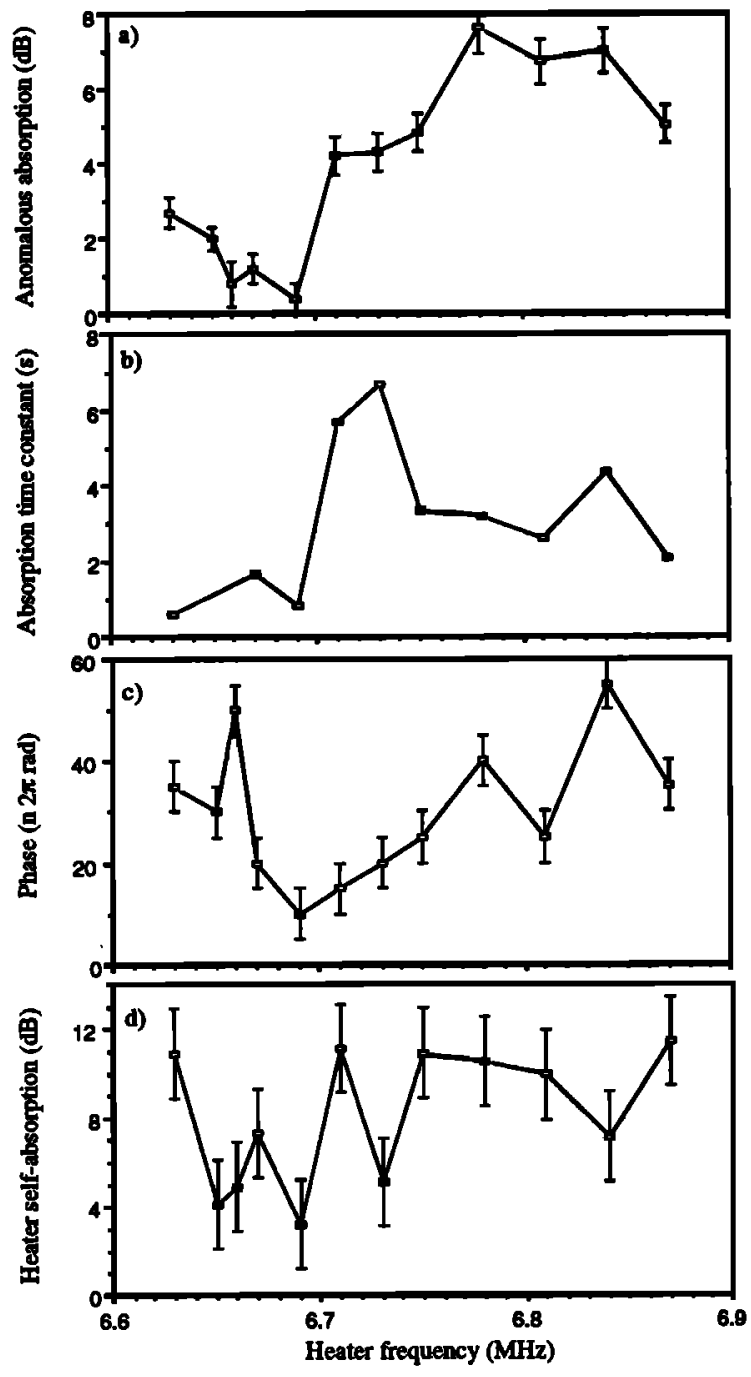

Fig. 7. Panels as for Fig, 6 except measurements obtained on May 15, 1991 , near to the fifth gyrofrequency harmonic. Note that heater ERP $640 \mathrm{MW}$ during this experiment.

heater frequency in Figure $6 c$ and Figure 7c. The error bars in these figures represent the typical phase changes present during periods in which no heating took place. Previous observations of heater-induced diagnostic phase changes [Jones et al., 1982; Robinson, 1989] have revealed that the phase change is well correlated with anomalous absorption level independently of ionospheric conditions. However, while Figures $6 c$ and $7 c$ do not exhibit a detailed correlation with the anomalous absorption, in general, the phase change is smaller at pump frequencies in the region of the absorption minimum and larger elsewhere. This result is in agreement with Doppler measurements by Lobachevsky et al. [1992] who also found that the Doppler (i.e., phase or electron density) change was reduced when the heater frequency was close to the third harmonic of the gyrofrequency. It should be noted that during both campaigns reported in this paper, the natural phase variations were quite large, which made measurement of the heater-induced phase changes rather difficult and which could account for the lack of detailed correlation with anomalous absorption. Furthermore, it is likely that the two 30-s on/off heater cycles transmitted during the experiments of May 1991 (Figure 2) did not allow the heater-induced phase change to either saturate or fully recover from heating since previous 
measurements at Troms $\emptyset$ indicate that the phase changes exhibit an e-fold time of close to $40 \mathrm{~s}$ [Jones et al., 1982; Robinson, 1989]. It may be then that the electron density (i.e., phase) had not completely recovered from heating at one pump frequency before heating at the next frequency commenced.

\subsection{Heater Self-Absorption}

The so-called heater "overshoot" where an initially strong reflected pump signal is rapidly attenuated is a common feature of heating experiments at Tromsø [Fejer and Kopka, 1981; Jones et al., 1986; Robinson, 1989]. The rapid quenching of the beater signal in the first few seconds after switch on which leads to the "overshoot" effect is a result of the anomalous selfabsorption of the heater wave. It should be noted that the time resolution of the data presented in this paper $(\sim 31 \mathrm{~ms})$ was insufficient to observe the reduction in reflected heater signal due to the parametric decay instability which occurs during the first few milliseconds after heater switch on. The heater selfabsorption may be quantified as the difference between the signal level observed during the first few seconds after heater switch on and the quasi-steady level obtained after this time. However, there are a number of potential problems which must be considered when estimating the self-absorption in this manner. Firstly, if the beater is switched on during a naturally occurring deep fade, then the overshoot will not be present and therefore the absorption value cannot be calculated or will be much reduced from the "true" value. In the experiments reported here, there are only 1 or 2 pulses (Figure 2) at each heater frequency where the overshoot might be observed and hence the errors associated with the measurement of self-absorption are, in general, higher than those associated with the diagnostic absorption. Secondly, unlike the diagnostic signals, the vertically launched heater wave propagates through the heated $D$ region before being reflected in the $F$ region. However, $D$ region effects, e.g., collisional absorption, are likely to occur on time scales much shorter $(<0.1 \mathrm{~s})$ than the anomalous absorption in the $F$ region. For the experiments of the type described in this paper, the effect of the propagation of the heater wave through the $D$ region should be virtually independent of heater frequency in each, relatively narrow, frequency range. In addition, previous observations at Troms $\emptyset$ [Robinson, 1989] indicate that anomalous absorption in the $F$ region is considerably stronger than heater-induced $D$ region absorption.

Examples of heater self-absorption as a function of heater frequency are presented in Figures $6 d$ and $7 d$. While the minimum in self-absorption on May 16, 1991, is located at much the same frequency as for anomalous absorption (Figure $6 a$ ), it is much broader extending for some $70 \mathrm{kHz}$ compared to about $20 \mathrm{kHz}$. The asymmetry in frequency exhibited by the self-absorption is also interesting with the absorption below the minimum $\sim 10 \mathrm{~dB}$, while above, it is $\sim 15-20 \mathrm{~dB}$. This asymmetry does not appear to be the result of a change in the local ionospheric conditions and it is not observed for all of the experiments (e.g., Figure 7d). It is reasonable to conclude that the asymmetry in heater self-absorption is evidence in favor of an additional process taking place at pump frequencies above the absorption minimum which reduces the reflected heater signal without having any effect on the formation of FAI and hence the anomalous absorption of the diagnostic wave. The minimum in self-absorption observed on May 15, 1991, is also located at the same pump frequency as the diagnostic absorption. The weak "double dip" feature observed in the anomalous absorption (Figure $7 a$ ) is strongly present in the self-absorption (Figure $7 d$ ). As noted above, the level of selfabsorption measured above and below the absorption minimum on May 15 is essentially the same.

It is interesting to note that the quantities in Figures 6 and 7 appear to be unaffected by the heater power. This is not unexpected since the electric field produced by the Troms $\emptyset$ heater even at lower powers (ERP $\sim 200 \mathrm{MW}$ ) can exceed the excitation threshold for the thermal parametric instability which is responsible for the generation of the FAI.

\section{ESTIMATE OF ELECTRON GYROFREQUENCY FROM ANOMALOUS ABSORPIION MINIMUM}

Although it is clear that the minimum in anomalous absorption observed at Troms $\varnothing$ occurs when the pump frequency is close to a harmonic of the electron gyrofrequency, it is not certain whether the minimum occurs when the pump is exactly at the harmonic. In order to investigate this the electron gyrofrequency estimated from the anomalous absorption observations has been compared with that derived from the IGRF magnetic field model (Figure 8). In this figure the frequency at which the minimum in anomalous absorption occurred divided by the gyrofrequency harmonic number, $n$ (e.g., for $6.77 \mathrm{MHz}, \mathrm{n}=5$ ), has been plotted as a function of heater interaction height. Data from all the successful experiments of both campaigns have been included in the figure. The heater interaction height has been determined by ray tracing [Jones and Stephenson, 1975] through the appropriate electron density profile. The ambient real-height electron density profiles have been calculated from ionograms by utilizing the POLAN computer code [Titheridge, 1985 , 1988]. The solid curve in Figure 8 represents the electron gyrofrequency $f_{c}$ calculated from the following relation, $f_{c}=e B / 2 \pi m_{e}$, where $e$ and $m_{e}$ are the electron charge and mass, respectively, and $B$ is the magnetic field strength derived from the IGRF 1990 model extrapolated to 1991. Observations of

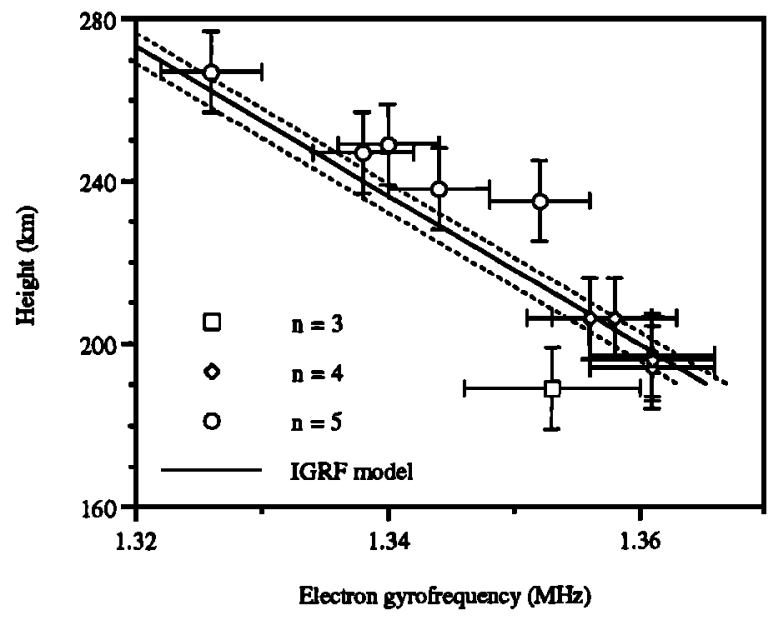

Fig. 8. Electron gyrofrequency as a function of height derived from anomalous absorption observations and the International Geomagnetic Reference Field (IGRF) 1990 magnetic field model extrapolated to 1991. The values derived from different gyrofrequency harmonics (i.e., $\mathrm{nf}_{\mathrm{c}}$ ) are marked with the various symbols indicated. The dashed lines represent values of the magnetic field separated from the IGRF model values by $\pm 70 \mathrm{nT}$. The value of $70 \mathrm{nT}$ represents the largest variation in the magnetic field encountered during the experiments for which data are plotted. 
the magnetic field at two European Incoherent Scatter (EISCAT) cross magnetometer stations [Luihr et al., 1984] have been examined. The station at Kilpisjärvi (KIL) $\left(-1.5^{\circ}\right.$ E of Troms $\left.\varnothing\right)$ measured a field which was typically $120-160 \mathrm{nT}$ below that predicted by the IGRF model, while the station at Alta (ALT) (nearly $4^{\circ} \mathrm{E}$ of Troms $\emptyset$ ) observed values about $10-60 \mathrm{nT}$ less than the IGRF model. The fields measured by the two stations generally varied by less than $70 \mathrm{nT}$ during the course of an experiment. However, it must be stressed that these magnetometers, while close to the heater site, are not on the same line of geomagnetic longitude as the heater and therefore the data must be interpreted with care. Examination of Figure 8 reveals a reasonable agreement between experiment and model which suggests that the minimum in the anomalous absorption is indeed related to the electron gyrofrequency harmonics. However, there are some large discrepancies between observation and the IGRF model which cannot entirely be explained by natural fluctuations in the magnetic field (dashed lines). Since the real-height density profile obtained from ionograms depends on a number of assumptions, especially about the lower ionosphere, it is possible that the errors in the reflection heights could be larger than those indicated in Figure 8. Furthermore, it should be noted that the reflection heights were interpolated from those obtained from the closest ionograms. For some of the data in Figure 8, ionograms were only obtained some time before and after the time when the absorption minimum occurred which could therefore lead to further errors. The large errors in the measurements, especially in the interaction height, make it difficult to determine whether the minimum occurs exactly at the harmonic.

\section{Discussion}

It is generally accepted that the anomalous absorption of HF probe waves is a result of scattering from FAI generated by an 0 mode pump wave through the action of the thermal parametric instability [e.g., Graham and Fejer, 1976]. Therefore a possible explanation for the reduction of anomalous absorption in the vicinity of electron gyrofrequency harmonics is that the FAI are only weakly excited by the heater at these pump frequencies. This interpretation is also supported by the disappearance of the DM from the SEE spectra since Leyser et al. [1992] argue that this feature is dependent on the generation of upper hybrid waves (UH) in the presence of FAI. However, although Fialer [1974] found an increase in field-aligned scattering cross section at pump frequencies close to the second harmonic, to our knowledge no radar observations of FAI intensity at pump frequencies close to highergyrofrequency harmonics have been reported. It should be noted that Leyser et al. [1992] found that the DM was absent in a limited range of heater frequencies, the exact range. being dependent on the gyrofrequency harmonic ( $5 \mathrm{kHz}$ for $\mathrm{n}=4,3$ $\mathrm{kHz}$ at $\mathrm{n}=5$, and $1 \mathrm{kHz}$ at $\mathrm{n}=6$ ). These authors also found that the intensity of the DM was weakened at pump frequencies up to $20 \mathrm{kHz}$ from the gyrofrequency harmonic. During the experiments reported here, the smallest step in heater frequency was $10 \mathrm{kHz}$ and therefore our measurements may not have been sufficiently close to the harmonic for anomalous absorption to be reduced to zero. However, it is clear that the maximum reduction in anomalous absorption occurs over a $20-\mathrm{kHz}$ range of pump frequencies.

The disappearance of the DM feature close to harmonics of the electron gyrofrequency has been treated in the literature by
Rao and Kaup [1990] who have developed a theory for upper hybrid wave conversion into nonpropagating electrostatic electron cyclotron harmonic (Bernstein) modes for electromagnetic waves close to harmonics of the electron gyrofrequency, whereas Leyser et al. [1990] have attributed the absence of the DM feature to the damping of the UH waves which are propagating with frequencies near harmonics of the electron gyrofrequency. In both cases, UH waves are required in the first place before either becoming mode converted or damped, whereas the excitation of the UH wave itself depends on the presence of FAI. On the other hand, the quenching of the anomalous absorption at frequencies close to harmonics of the electron gyrofrequency indicates that the intensity of the FAI is probably reduced at these frequencies, implying that the UH waves may only weakly be excited in the first place. We therefore suggest that other mechanisms such as direct conversion of electromagnetic waves into electrostatic Bernstein modes are involved. A detailed calculation regarding the required threshold value for the suggested process is beyond the scope of this paper and will be dealt with elsewhere.

The heater self-absorption is also reduced for pump frequencies close to electron gyrofrequency harmonics. The higher level of heater self-absorption at pump frequencies above the gyrofrequency harmonic compared to those observed below (Figure 6) is not accompanied by a similar asymmetry in the other measured quantities (i.e. anomalous absorption, absorption onset time, and phase changes). This would seem to indicate that the mechanism leading to additional heater absorption which operates above the gyrofrequency does not result in the intensification of FAI (and hence increased anomalous absorption) or enhanced large-scale (i.e., phase) effects. It is interesting to note that in the experiments performed to date, the asymmetry in heater self-absorption has always been observed at the fourth harmonic of the gyrofrequency but never at either the third or the fifth harmonics. This suggests that the mechanism which leads to the asymmetry in self-absorption requires the relatively stable heater reflection height found for pump frequencies well below the $F$ region peak and well above the $E$ region.

The "double dip" which is always observed in anomalous absorption (e.g., Figure 4) at the fifth harmonic and occasionally at the fourth harmonic is also seen in the heater self-absorption. This feature is also observed when the data are averaged over a number of experiments (Figure 5) and therefore would appear to be a systematic feature. Currently, there appears to be no theoretical explanation for the presence of the "double dip."

Acknowledgments. Two of us (F.H. and A.J.S.) were supported in this work by S.E.R.C. grants GRF80784 and GRH32025. We would like to thank H. Gegner for operating the heater and the Leicester technicians (M. Parsons, M. Popple, P. Chapman, and B. Dhanda) for their stoicism in the face of what were long and demanding experiments. Thanks are also due to J. Morey for her assistance in deriving the anomalous absorption growth times.

The Editor thanks T. Leyser and B. Lundborg for their assistance in evaluating this paper.

\section{REFERENCES}

Belyakova, V. N., et al., The features of excitation of plasma turbulence by a high-power radio wave field under even-order gyroresonance conditions, Geomagn. Aeron., 31, 367-370, 1991.

Cohen, R., and J.D. Whitehead, Radio-reflectivity detection of artificial modification of the ionospheric F layer, J. Geophys. Res., 75, 6439. 6445, 1970. 
Das, A.C., and J.A. Fejer, Resonance instability of small-scale fieldaligned irregularities, J. Geophys. Res., 84, 6701-6704, 1979.

Dysthe, K.B., E. Mjølhus, H.L. Pesceli, and K. Rypdal, A thermal oscillating two-stream instability, Phys. Fluids, 26, 146-157, 1983.

Fejer, J.A., and H. Kopka, The effects of plasma instabilities on the ionospherically reflected wave from a high power transmitter, $J$. Geophys. Res., 86, 5746-5750, 1981.

Fejer, J.A., C.A. Gonzales, H.M. Ierkic, M.P. Sulzer, C.A. Tepley, L.M Duncan, F.T. Djuth, S. Ganguly, and W.E. Gordon, Ionospheric modification experiments with the Arecibo heating facility, J. Atmos. Terr. Phys., 47, 1165-1179, 1985.

Fialer, P.A., Field-aligned scattering from a heated region in the ionosphere - Observations at HF and VHF, Radio Sci., 9, 923-940, 1974.

Graham, K.N., and J.A. Fejer, Anomalous radio wave absorption due to ionospheric heating effects, Radio Sci., 11, 1057-1063, 1976.

Jones, R.M., and J.J. Stephenson, A versatile 3D ray tracing computer program for radio waves in the ionosphere, Rep. OT75-76, U.S. Dep. of Commerce, Washington, D.C., 1975.

Jones, T.B., T. Robinson, P. Stubbe, and H. Kopka, Phase changes induced in a diagnostic radio wave passing through a heated region of the auroral ionosphere, J. Geophys. Res., 87, 1557-1564, 1982.

Jones, T.B., T.R. Robinson, P. Stubbe, and H. Kopka, Frequency dependence of anomalous absorption caused by high power radio waves, J. Atmos. Terr. Phys., 46, 147-153, 1984.

Jones, T.B., T.R. Robinson, P. Stubbe, and H. Kopka, EISCAT observations of the heated ionosphere, J. Atmos. Terr. Phys., 48, 1027-1035, 1986.

Kuo, S.P., and M.C. Lee, On parametric excitation of plasma modes at upper-hybrid resonance, Phys. Lett. A, 91, 444446, 1982.

Leyser, T.B., Parametric interaction between upper hybrid and lower hybrid waves in heating experiments, Geophys. Res. Lett., 18, 408411, 1991.

Leyser, T.B., B. Thide, H. Derblom, A. Hedberg, B. Lundborg, P. Stubbe, and H. Kopka, Stimulated electromagnetic emission near electron cyclotron harmonics in the ionosphere, Phys. Rev. Lett., 63, 1145 . $1147,1989$.

Leyser, T.B., B. Thide, H. Derblom, A. Hedberg, B. Lundborg, P. Stubbe, and H. Kopka, Dependence of stimulated electromagnetic emission on the ionosphere and pump wave, J. Geophys. Res., 95, 17,233$17,244,1990$.

Leyser, T.B., B. Thide, S. Goodman, M. Waldenvik, E. Veszelei, S.M. Grach, A.N. Karashtin, G.P. Komrakov, and D.S. Kotik, Narrow cyclotron harmonic absorption resonances of stimulated electromagnetic emission in the ionosphere, Phys. Rev. Lett., 68, 3299-3302, 1992.

Lobachevsky, L.A., Yu.V. Gnuzdev, V.Yu. Kim, G.A. Mikhaylova, V.A. Panchenko, V.P. Polimatidi, V.A. Puchkov, V.V. Vaskov, P. Stubbe, and H. Kopka, Observations of ionospheric modification by the Troms heating facility with the mobile diagnostic equipment of IZMIRAN, J. Atmos. Terr. Phys., 54, 75-85, 1992.

Lôhr, H., S. Thürey, and N. Klöcker, The EISCAT-magnetometer cross : Operational aspects - first results, Geophys. Surv., 6, 305-315, 1984.

Perkins, F.W., A theoretical model for short-scale field-aligned plasma density striations, Radio Sci., 9, 1065-1070, 1974.

Rno, N.N., and D.J. Kaup, Upper hybrid mode conversion and resonance excitation of Bernstein modes in ionospheric heating experiments, $J$. Geophys. Res., 95, 17,245-17,252, 1990.

Robinson, T.R., The excitation of plasma waves and irregularities in the ionosphere by means of high power radio waves, Plasma Phys. Controlled Fusion, 30, 45-56, 1988.

Robinson, T.R., The heating of the high latitude jonosphere by high power radio waves, Phys. Rep., 179, 79-209, 1989.

Ryzhov, Yu. A., Thermal radiation in a randomly inhomogeneous medium, Sov. Phys. JETP, Engl. Transl., 32, 120-124, 1971.

Ryzhov, Yu. A., Absorption of the energy of an electromagnetic field in a randomly inhomogeneous plasma, Sov. Phys. JETP, Engl. Transl., 35, 490-493, 1972.

Stocker, A.J., T.R. Robinson, and T.B. Jones, Observations of the effects of ionospheric heating on the amplitude of low power diagnostic radio waves at Arecibo, J. Geophys. Res., 97, 6315-6322, 1992a.

Stocker, A.J., F. Honary, T.R. Robinson, T.B. Jones, P. Stubbe, and H. Kopka, EISCAT observations of large scale electron temperature and electron density perturbations caused by high power HF radio waves, J. Atmos. Terr. Phys., 54, 1555-1572, $1992 b$.

Stubbe, P., and H. Kopka, Modification of the F-region by powerful radio waves, in Exploration of the polar Upper Atmosphere, edited by C.S. Deehr and J.A. Holtet, p. 83, D. Reidel, Norwell, Mass., 1980.

Stubbe, $P$., and H. Kopka, Stimulated electromagnetic emissions in a magnetized plasma: A new symmetric spectral feature, Phys. Rev. Lett., 65, 183-186, 1990.

Stubbe, P., H. Kopka, T.B. Jones, and T. Robinson, Wide band attenuation of radio waves caused by powerful HF waves: Saturation and dependence of ionospheric variability, J. Geophys. Res., 87, 1551 - 1555, 1982a.

Stubbe, P., et al., Ionospheric modification experiments in northern Scandinavia, J. Atmos. Terr. Phys., 44, 1025-1041, $1982 b$

Stubbe, P., H. Kopka, B. Thidé, and H. Derblom, Stimulated Electromagnetic Emission: A New technique to study the parametric decay instability in the ionosphere, J. Geophys. Res., 89, 7523-7536, 1984.

Thide, B., H. Kopka, and P. Stubbe, Observations of stimulated scattering of a strong high-frequency radio wave in the ionosphere, Phys. Rev. Lett., 49, 1561-1564, 1982.

Thidé, B., H. Derblom, A. Hedberg, H. Kopka, and P. Stubbe, Observations of stimulated electromagnetic emissions in ionospheric heating experiments, Radio Sci., 18, 851-859, 1983.

Thide, B., A. Hedberg, J.A. Fejer, and M.P. Sulzer, First Observations of stimulated electromagnetic emission at Arecibo, Geophys. Res. Lett., 16, 369-372, 1989.

Titheridge, J.E., Ionogram analysis with the generalized program POLAN, Rep. UAG-93, pp 1-194, World Data Cent. A for Sol.-Terr. Phys., NOAA, E/GC2, Boulder, Colo., 1985.

Titheridge, J.E., The real height analysis of ionograms: A generalized formulation, Radio Sci., 23, 831-849, 1988.

Utlaut, W.F., and E.J. Violette, A summary of vertical incidence radio observations of ionospheric modifications, Radio Sci., 9, 895-903, 1974.

Vas'kov, V.V., and A.V . Gurevich, Nonlinear resonant instability of plasma in the reflection region of an ordinary electromagnetic wave, Sov. Phys. JETP, Engl. Transl., 42, 91-97, 1975.

Vas'kov, V.V., and A.V. Gurevich, Resonance instability of small scale perturbations, Sov. Phys. JETP, Engl. Transl., 46, 487-494, 1977.

F. Honary, T.B. Jones, T.R. Robinson, and A.J. Stocker, Department of Physics and Astronomy, Leicester University, University Road, Leicester, LE1 7RH, England.

P. Stubbe, Max-Planck-Institut für Aeronomie, Lindau, Germany.

(Received November 9, 1992; revised March 2, 1993; accepted March 29, 1993.) 